\title{
Resonant Effect for Breit-Wheeler Process in the Field of an X-ray Pulsar
}

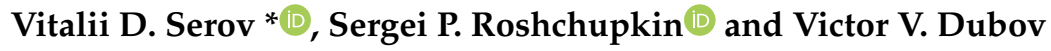 \\ Department of Theoretical Physics, Peter the Great St. Petersburg Polytechnic University, Polytechnicheskaya 29, \\ 195251 Saint Petersburg, Russia; roshchupkin_s@spbstu.ru (S.P.R.); dubov@spbstu.ru (V.V.D.) \\ * Correspondence: vitalii_serov@inbox.ru
}

Received: 13 September 2020; Accepted: 20 October 2020; Published: 22 October 2020

\begin{abstract}
The resonant process of the creation of an ultrarelativistic electron-positron pair by two hard gamma quanta in the field of an X-ray pulsar (the Breit-Wheeler process modified by an external field) was theoretically studied. Under resonance conditions, the intermediate virtual electron (positron) in the external field becomes a real particle. As a result, there are four reaction channels for the process instead of two. For each of those channels, the initial process of the second order in the fine structure constant in the field of an X-ray pulsar effectively reduces into two successive processes of the first order: X-ray-stimulated Breit-Wheeler process and X-ray-stimulated Compton effect. The resonant kinematics of the process was also studied in detail. The process had characteristic threshold energy, and all initial and final particles had to be ultrarelativistic and propagate in a narrow cone. Furthermore, the resonant energy spectrum of the electron-positron pair significantly depended on emission angles. Clearly, there was a qualitative difference between resonant and nonresonant cases. Lastly, the resonant differential probability of studied process was obtained. The resonant differential probability significantly exceeded the nonresonant one without the external field of an X-ray pulsar.
\end{abstract}

Keywords: external field QED; Oleinik's resonances; Breit-Wheeler process; X-ray pulsars

\section{Introduction}

Astroparticle physics is currently a rapidly developing and relevant field of investigation [1] Quantum processes also play a significant role in the evolution of astrophysical objects such as pulsars and magnetars due to the presence of a strong electromagnetic field [2]. For example, anomalous high-energy positron abundance in cosmic rays was observed, and there is an assumption about their origin from pulsars [3,4]. Several effects of quantum electrodynamics (QED) in external fields can reproduce the production of electron-positron pairs due to interactions of gamma quanta with the electromagnetic field of a pulsar [5,6].

In 1967, V. Oleinik discovered a resonant mode in second-order (or higher) external-field QED processes $[7,8]$ (also see reviews $[9,10])$. Oleinik resonances correspond to an intermediate virtual state coming on a mass shell and becoming a real particle. In this case, the initial second-order process effectively reduces into two successive processes of the first order because lower-order processes are allowed in the external field. The probability of that kind of process is greatly increased in comparing to nonresonant ones. Moreover, the energies of the final particles strongly depends on their outgoing angles. The study of resonant QED processes in an external field has had high priority in the last 30 years [11-20].

External-field QED processes connected with the development of high-power laser technologies are discussed in [21] in the context of the potential discovery of new particles beyond the standard model. The conceptual developments of fundamental QED processes, which followed the basic work of 
Dirac and Breit-Wheeler, were analyzed and turned into astrophysics in [22]. In [23], the nonresonant Breit-Wheeler pair production by a probe photon traversing two consecutive short and ultrashort laser pulses was studied. In [24], energy-angular distributions of electron-positron pair creation in collisions of a laser beam and a nonlaser photon were calculated using S-matrix formalism. The resonant Breit-Wheeler process in the field of a plane monochromatic electromagnetic wave was analysed in [25], but the resonant kinematics and differential probability of the process were not studied in detail. In [26], the process of electron-positron annihilation into two photons in the presence of an intense classical plane wave of an arbitrary shape was analytically investigated by employing light-cone quantization and by exactly considering the effects of the plane wave.

In contrast to above-mentioned works, in this paper, the resonant Breit-Wheeler process is studied in the field of an X-ray pulsar. The resonant kinematics of the process is mostly on focus, as well as a comparison of differential probabilities in resonant and nonresonant cases.

There is a characteristic parameter for processes in the external field called the classical relativistic-invariant parameter [27] (natural units $c=\hbar=1$ are used):

$$
\eta=\frac{e F}{m \omega}
$$

where $e$ and $m$ are the electron's charge and mass, $F$ is the strength of the field, and $\omega$ is the wave frequency.

According to [28,29], for an X-ray pulsar with $\omega \sim 1-100 \mathrm{keV}$ and $F \sim 10^{11}-10^{13} \mathrm{~V} / \mathrm{cm}$, the parameter takes a value of order of $\eta \lesssim 10^{-1}$. Therefore, electromagnetic fields are considered as weak in this paper, and the following condition is satisfied:

$$
\eta \ll 1
$$

\section{Amplitude of X-ray-Assisted Breit-Wheeler Process}

The field of a pulsar was assumed to be a monochromatic electromagnetic circularly polarized plane wave propagating along the $\mathrm{z}$ axis in the following form:

$$
A=\frac{F}{\omega}\left(e_{x} \cos \varphi+\delta e_{y} \sin \varphi\right), \quad \varphi=k x=\omega(t-z),
$$

where $A$ and $\omega$ are the 4-potential and frequency of the wave, $e_{x}=\left(0, \mathbf{e}_{\mathbf{x}}\right), e_{y}=\left(0, \mathbf{e}_{\mathbf{y}}\right), k=\omega(1, \mathbf{n})$ are the 4 -vectors of polarization and momentum of the wave, and $\delta= \pm 1$.

The production of an electron-positron pair by two gamma quanta in the external electromagnetic field is the second-order process in the fine structure constant, and it can be described in terms of two Feynman diagrams (see Figure 1).

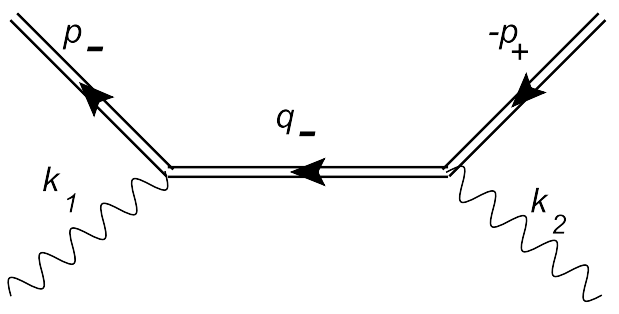

(a)

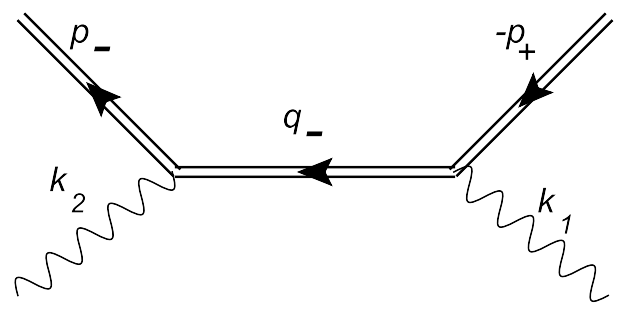

(b)

Figure 1. Feynman diagrams of electron-positron-pair production by two gamma $(\mathbf{a}, \mathbf{b})$ quanta in the field of an X-ray pulsar. External double lines correspond to Volkov functions of electron or positron, wavy lines correspond to wave functions of initial gamma quanta, and internal double lines correspond to Green's electron function in the field of a plane electromagnetic wave. 
The amplitude of the process can be written in the following form:

$$
S_{i f}=i e^{2} \iint d^{4} x_{1} d^{4} x_{2} \bar{\Psi}_{p_{-}}\left(x_{1} \mid A\right) \hat{A}_{1}\left(x_{1} ; k_{1}\right) G\left(x_{2} x_{1} \mid A\right) \hat{A}_{2}\left(x_{2} ; k_{2}\right) \Psi_{-p_{+}}\left(x_{2} \mid A\right)+\left(k_{1} \longleftrightarrow k_{2}\right),
$$

where $k_{1,2}=\omega_{1,2}\left(1, \mathbf{n}_{1,2}\right)$ are the 4-momenta of initial gamma quanta, $p_{ \pm}=\left(E_{ \pm}, \mathbf{p}_{ \pm}\right)$are the 4-momenta of the electron-positron pair, and

$$
\Psi_{p}(x)=\mathfrak{J}_{p}(x) \frac{U_{p}}{\sqrt{2 E}}, \quad \mathfrak{J}_{p}(x)=\left[1+\frac{e}{2(p k)} \hat{k} \hat{A}(k x)\right] e^{i S_{p}(x)}, \quad \hat{A} \equiv \gamma_{0} A^{0}-(\vec{\gamma}, \mathbf{A}),
$$

is the Volkov function of an electron (positron) [30,31], $U_{p}$ is free Dirac bispinor. Here, $\bar{\Psi}_{p}$ denotes the Dirac adjoint of $\Psi_{p}$, and index $-p_{+}$stands to emphasize that positron Volkov function can formally be obtained from the electron one by the sign changing of the 4-momentum.

Equation (5) also contains the classical action of an electron in the field of a monochromatic electromagnetic plane wave [32]:

$$
S_{p}(x)=-(p x)-\frac{e}{(k p)} \int_{0}^{k x} d \varphi\left[p A(\varphi)-\frac{e}{2} A^{2}(\varphi)\right] .
$$

Function $A_{j}$ in Equation (4) defines the 4-potentials of initial gamma quanta:

$$
A_{j}^{\mu}=\sqrt{\frac{2 \pi}{\omega_{j}}} \varepsilon_{j}^{\mu} e^{-i k_{j} x}, \quad j=1,2,
$$

where $\varepsilon_{j}^{\mu}$ are polarization 4 -vectors of initial gamma quanta.

Function $G\left(x_{2} x_{1} \mid A\right)$ in Equation (4) is the Green's function that describes the propagation of an intermediate electron within the external field. It can be written with the following integral [33,34]:

$$
G\left(x_{2} x_{1} \mid A\right)=\int \frac{d^{4} p}{(2 \pi)^{4}} \mathfrak{J}_{p}\left(x_{2}\right) \frac{\hat{p}+m}{p^{2}-m^{2}} \overline{\mathfrak{J}}_{p}\left(x_{1}\right) .
$$

After substitutions of Equations (5)-(8) into Equation (4) and integrations, the amplitude of the process in the case of weak-field Equation (2) takes the form

$$
S_{i f}=\sum_{l=-\infty}^{+\infty} S_{l}
$$

where $S_{l}$ is a partial amplitude of the process with the radiation or absorption of $|l|$-photons of the external electromagnetic field:

$$
S_{l}=\frac{i \pi e^{2}(2 \pi)^{4} e^{i d}}{4 \sqrt{E_{-} E_{+} \omega_{1} \omega_{2}}} \times\left(\bar{U}_{p_{-}} M_{l} U_{-p_{+}}\right) \times \delta^{(4)}\left(k_{1}+k_{2}-p_{-}-p_{+}+l k\right),
$$

where $\mathrm{d}$ is independent of the summation index phase.

Here is denoted

$$
\begin{gathered}
M_{l}=\sum_{l_{1}=-\infty}^{+\infty}\left(\varepsilon_{1}\right)_{\mu}\left[H_{l_{1}}^{\mu}\left(q_{-}, p_{-}\right) \frac{\hat{q}_{-}+m}{q_{-}^{2}-m^{2}} K_{l-l_{1}}^{v}\left(q_{-}, p_{+}\right)+H_{l_{1}}^{\mu}\left(q_{-}, p_{+}\right) \frac{\hat{q}_{-}+m}{q_{-}^{2}-m^{2}} K_{l-l_{1}}^{v}\left(q_{-}, p_{-}\right)\right]\left(\varepsilon_{2}\right)_{v}, \\
q_{-}=k_{2}-p_{+}+l_{1} k \quad \text { or } \quad q_{-}=k_{1}-p_{+}+l_{1} k .
\end{gathered}
$$

The first term in Equation (11) corresponds to reaction channel (a) and the second corresponds to channel (b). With definition $e_{ \pm} \equiv e_{x} \pm i e_{y}$ : 


$$
\begin{aligned}
& H_{l_{1}}^{\mu}\left(p^{\prime}, p\right)=\gamma^{\mu} L_{l_{1}}\left(\gamma_{p^{\prime}}, \chi_{p^{\prime} p}\right)+\frac{m \eta}{4}\left(\frac{\hat{e}_{-} \hat{k} \gamma^{\mu}}{\left(p^{\prime} k\right)}-\frac{\gamma^{\mu} \hat{e}_{-} \hat{k}}{(p k)}\right) L_{l_{1}-1}+\frac{m \eta}{4}\left(\frac{\hat{e}_{+} \hat{k} \gamma^{\mu}}{\left(p^{\prime} k\right)}-\frac{\gamma^{\mu} \hat{e}_{+} \hat{k}}{(p k)}\right) L_{l_{1}+1} \\
& K_{l-l_{1}}^{\nu}\left(p^{\prime}, p\right)=\gamma^{v} L_{l-l_{1}}\left(\gamma_{p^{\prime} p}, \chi_{p^{\prime} p}\right)+\frac{m \eta}{4}\left(\frac{\hat{e}_{-} \hat{k} \gamma^{v}}{\left(p^{\prime} k\right)}+\frac{\gamma^{v} \hat{e}_{-} \hat{k}}{(p k)}\right) L_{l-l_{1}-1}+\frac{m \eta}{4}\left(\frac{\hat{e}_{+} \hat{k} \gamma^{v}}{\left(p^{\prime} k\right)}+\frac{\gamma^{v} \hat{e}_{+} \hat{k}}{(p k)}\right) L_{l-l_{1}+1} .
\end{aligned}
$$

Moreover, in the case of circular polarization, special functions $L_{n}$ can be written with Bessel functions of an integer index [35]:

$$
L_{n}\left(\gamma_{p^{\prime} p}, \chi_{p^{\prime} p}\right)=\exp \left(-i n \chi_{p^{\prime} p}\right) J_{n}\left(\gamma_{p^{\prime} p}\right),
$$

and their arguments are defined in the following form:

$$
\gamma_{p^{\prime} p}=m \eta \sqrt{-Q_{p^{\prime} p^{\prime}}^{2}}, \quad \tan \chi_{p^{\prime} p}=\delta \frac{\left(Q_{p^{\prime} p} e_{y}\right)}{\left(Q_{p^{\prime} p} e_{x}\right)}, \quad Q_{p^{\prime} p}=\frac{p^{\prime}}{\left(p^{\prime} k\right)}-\frac{p}{(p k)} .
$$

\section{Resonant Kinematics}

Resonance is possible only due to the presence of the external field. Then, the intermediate electron or positron with 4-momentum $q_{-}$or $q_{+}$comes to mass shell

$$
q_{ \pm}^{2}=m^{2}
$$

therefore, amplitude Equation (10) tends to infinity.

Under the resonance condition, the process effectively reduces into two successive processes for each channel: X-ray-stimulated Breit-Wheeler process and X-ray-stimulated Compton effect. There are four reaction channels (see Figure 2).

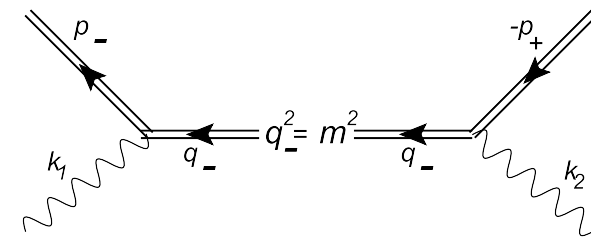

(a)

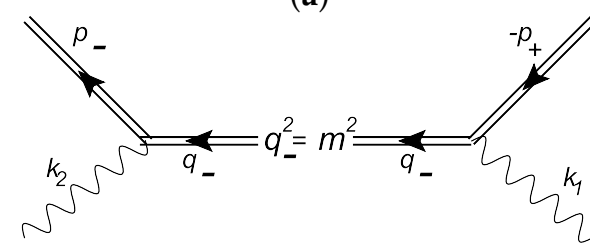

(b)

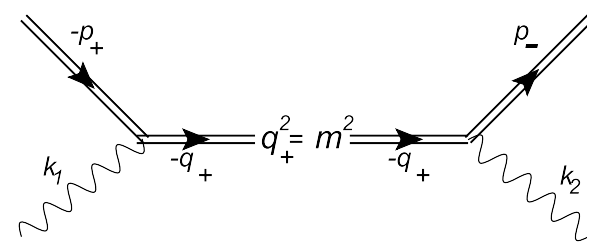

(c)

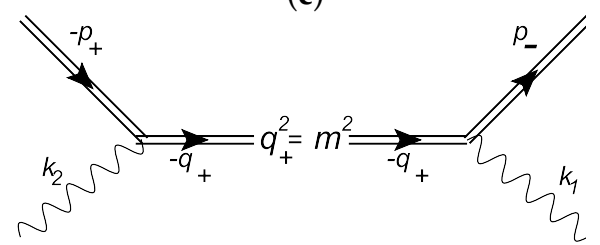

(d)

Figure 2. Feynman diagrams of resonant electron-positron pair production by two gamma quanta in the field of an X-ray pulsar. There is an electron intermediate state for channels $(\mathbf{a}, \mathbf{b})$, and a positron one for channels $(\mathbf{c}, \mathbf{d})$.

It is sufficient to only consider kinematics of channel (a); the three other channels are obtained from that by index changing. The most probable case is the process with radiation or absorption of only one photon of the external wave in both vertices $\left(l_{1}=1\right.$ and $l=0$ in Equation (11)) due to the weak field under Equation (2).

Henceforth, initial gamma quanta and final electron-positron pair are considered to be ultrarelativistic:

$$
\omega_{1,2} \gg m, \quad E_{ \pm} \gg m .
$$


Conditions in Equation (18) are necessary for resonant reaction channels with Equation (17) due to the presence of threshold value $\omega_{t h r}$ for the initial gamma quanta energies (see Equations (22)-(23) and Equation (34) below). That threshold energy can be estimated for an X-ray pulsar with the order of $\omega_{t h r} \sim 10^{2} \mathrm{MeV}$ (see the text after Equation (23)) and with corresponding conservation laws (see Equation (20) and (26) below) leads to conditions Equation (18).

In a considerable case, all particles propagate in a narrow cone, the axis of which is far away from the direction of propagation of the X-ray wave. Oleinik resonances are gone with no satisfaction of the last condition. According to this, the angles for all particles are defined as follows:

$$
\theta_{j \pm} \equiv \angle\left(\mathbf{k}_{j}, \mathbf{p}_{ \pm}\right) \ll 1, \quad \theta_{j} \equiv \angle\left(\mathbf{k}_{j}, \mathbf{k}\right) \sim 1, \quad j=1,2 ; \quad \theta_{ \pm} \equiv \angle\left(\mathbf{p}_{ \pm}, \mathbf{k}\right) \sim 1
$$

So, resonance for channel (a) is considered. Starting from the 4-momentum conservation law in vertex 2 (X-ray-stimulated Breit-Wheeler process):

$$
p_{+}+q_{-}=k_{2}+k
$$

Using conditions in Equations (17)-(19), one can obtain the equation for positron energy:

$$
4\left(\varepsilon_{2}+\frac{\varepsilon_{2}^{2} \delta_{2+}^{2}}{\varepsilon_{i}^{2}}\right) X_{+}^{2}-4 \frac{\varepsilon_{2}^{2}}{\varepsilon_{i}} X_{+}+\frac{\varepsilon_{2}^{2}}{\varepsilon_{i}^{2}}=0,
$$

which are denoted

$$
\omega_{i} \equiv \omega_{1}+\omega_{2}, \quad X_{+} \equiv \frac{E_{+}}{\omega_{i}}, \quad \varepsilon_{2} \equiv \frac{\omega_{2}}{\omega_{t h r}}, \quad \varepsilon_{i}=\frac{\omega_{i}}{\omega_{t h r}}, \quad \omega_{t h r} \equiv \frac{m^{2}}{\omega \sin ^{2} \frac{\theta_{2}}{2}}, \quad \delta_{2+} \equiv \frac{\omega_{i}}{2 m} \theta_{2+} .
$$

Here, $\omega_{t h r}$ is the threshold energy for the X-ray-stimulated Breit-Wheeler process. It appears from the discriminant of Equation (21):

$$
D=16 \varepsilon_{2}^{4} \varepsilon_{i}^{-2}\left[1-\varepsilon_{2}^{-1}-\varepsilon_{i}^{-2} \delta_{2+}^{2}\right] \geq 0 \Longrightarrow \varepsilon_{2} \geq 1 \Longleftrightarrow \omega_{2} \geq \omega_{t h r}
$$

One can simply estimate an order of the threshold energy for an X-ray pulsar. For instance, the energy of X-ray gamma quantum $\omega=2.5 \mathrm{keV}$ and angle $\theta_{2}=\pi$ leads to $\omega_{t h r}=100 \mathrm{MeV}$, so that the value of threshold energy was of the order of $10^{2} \mathrm{MeV}$ for calculations in this paper.

Equation (21) has two solutions to positron energies:

$$
X_{+1,2}=\frac{1}{2 \varepsilon_{i} \beta_{2+}}\left[1 \pm \sqrt{1-\beta_{2+}}\right], \quad \beta_{2+} \equiv \frac{1}{\varepsilon_{2}}+\frac{\delta_{2+}^{2}}{\varepsilon_{i}^{2}} .
$$

that also leads to the restriction for outgoing positron angle:

$$
0 \leq \delta_{2+}^{2} \leq \frac{\varepsilon_{i}^{2}\left(\varepsilon_{2}-1\right)}{\varepsilon_{2}} \equiv \delta_{2 \max }^{2}
$$

Maximal value $\delta_{2 \max }^{2}$ depends on the energies of initial gamma quanta.

The next step is consideration of the X-ray-stimulated Compton effect in vertex 1 . Writing corresponding 4-momentum conservation law

$$
k_{1}+q_{-}=p_{-}+k
$$

and again using Equations (17)-(19), one can obtain the equation for electron energy:

$$
4\left(\varepsilon_{1}-\frac{\varepsilon_{1}^{2} \delta_{1-}^{2}}{\varepsilon_{i}^{2}}\right) X_{-}^{2}-4 \frac{\varepsilon_{1}^{2}}{\varepsilon_{i}} X_{-}-\frac{\varepsilon_{1}^{2}}{\varepsilon_{i}^{2}}=0 .
$$


Here, denoted are

$$
X_{-} \equiv \frac{E_{-}}{\omega_{i}}, \quad \varepsilon_{1} \equiv \frac{\omega_{1}}{\omega_{t h r}}, \quad \delta_{1-} \equiv \frac{\omega_{i}}{2 m} \theta_{1-} .
$$

Equation (27) has only one physical solution:

$$
X_{-}=\frac{1}{2 \varepsilon_{i} \beta_{1-}}\left[1+\sqrt{1+\beta_{1-}}\right]<1, \quad \beta_{1-} \equiv \frac{1}{\varepsilon_{1}}-\frac{\delta_{1-}^{2}}{\varepsilon_{i}^{2}}
$$

and it leads to a restriction for the outgoing electron angle:

$$
0 \leq \delta_{1-}^{2}<\frac{\varepsilon_{i}^{2}}{\varepsilon_{1}} \equiv \delta_{1 \max }^{2}
$$

Maximal value $\delta_{1 \text { max }}^{2}$ depends on the energies of the initial gamma quanta, but the corresponding angle must also not be near that maximum due to physical condition $X_{-}<1$.

So, there are two possible values for the energy of positron given by Equation (24). However, there is only one possible value for electron energy given by Equation (29), but there must be two values, too, according to the overall energy-conservation law, which takes the following form in weak-field Equation (2):

$$
\omega_{i} \approx E_{+}+E_{-} \Longrightarrow X_{-}=1-X_{+}
$$

As a result, electron energy can be written as

$$
X_{-}=1-\frac{1}{2 \varepsilon_{i} \beta_{2+}}\left[1 \pm \sqrt{1-\beta_{2+}}\right]
$$

Of course, Equations (29) and (32) must be agree and have points of intersection. One way for this to be possible is using the following assumption:

$$
\delta_{1-}^{2}=\delta_{2+}^{2} \equiv \delta_{* \prime}^{2}
$$

which is illustrated by graphs (see Figure 3).

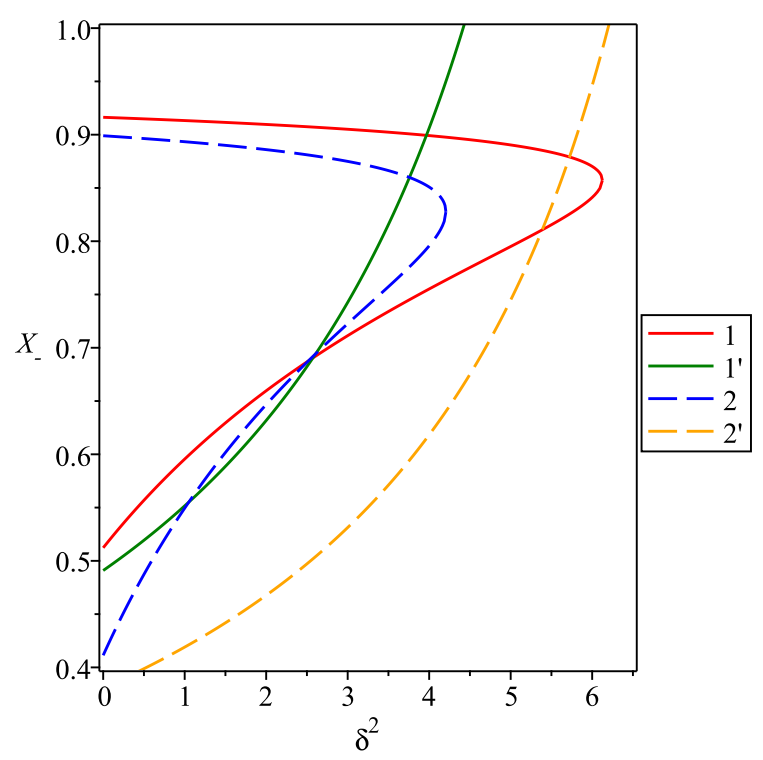

Figure 3. Electron energies. Variable $\delta^{2}$ corresponds to $\delta_{2+}^{2}$ or $\delta_{1-}^{2}$. Lines 1 and 2 correspond to Equation (32), lines $1^{\prime}$ and $2^{\prime}$ correspond to Equation (29). $\varepsilon_{1}=1.5, \varepsilon_{2}=2$ for lines 1 and $1^{\prime}$, there are two points of intersection $\delta_{*}^{2} . \varepsilon_{1}=0.9, \varepsilon_{2}=2$ for lines 2 and $2^{\prime}$, there are no points of intersection. 
Moreover, analysis showed (in agreement with [25]) that Equations (29) and (32) can be solved together if only

$$
\varepsilon_{1} \geq 1, \quad \varepsilon_{2} \geq 1,
$$

thus, both initial gamma quanta must have minimal energies of $\omega_{t h r}$.

One can define the values for angles of intersection $\delta_{*}^{2}$ from the following transcendental equations:

$$
\frac{1}{2 \varepsilon_{i} \beta_{1-}\left(\delta_{*}^{2}\right)}\left[1+\sqrt{1+\beta_{1-}\left(\delta_{*}^{2}\right)}\right]=1-\frac{1}{2 \varepsilon_{i} \beta_{2+}\left(\delta_{*}^{2}\right)}\left[1 \pm \sqrt{1-\beta_{2+}\left(\delta_{*}^{2}\right)}\right] .
$$

Results must agree with both of conditions given by Equations (25) and (30).

\section{Resonant Kinematics for High Initial Gamma Quanta Energy}

In this section, initial gamma quanta are considered to have energies that extremely exceeds threshold energy, and the following conditions are satisfied:

$$
\varepsilon_{1}<\varepsilon_{2} \gg 1
$$

Additionally, parameters $\beta_{2+}$ and $\beta_{1-}$ were assumed to be small. This assumption led to some restrictions on outgoing electron and positron angles:

$$
\begin{gathered}
\delta_{2+}^{2} \ll \varepsilon_{i}^{2} \Longrightarrow \beta_{2+} \ll 1, \\
\delta_{1-}^{2} \ll \frac{\varepsilon_{i}^{2}}{\varepsilon_{1}} \sim \varepsilon_{1} \Longrightarrow \beta_{1-} \ll 1 .
\end{gathered}
$$

Using Equations (36)-(38), one can expand solutions to positron and electron energies with Equations (24) and (29) to obtain:

$$
\begin{aligned}
& X_{+} \approx\left(\varepsilon_{i} \beta_{2+}\right)^{-1}\left(1-\frac{1}{4} \beta_{2+}\right), \\
& X_{-} \approx\left(\varepsilon_{i} \beta_{1-}\right)^{-1}\left(1+\frac{1}{4} \beta_{1-}\right) .
\end{aligned}
$$

The second solution to positron energy $\left(X_{+} \approx\left(4 \varepsilon_{i}\right)^{-1} \ll 1\right)$ was eliminated due to disagreement with energy-conservation law Equation (31).

Using overall energy-conservation law Equation (31), and Equation (40), positron energy can be written as follows:

$$
X_{+}=1-X_{-} \approx 1-\frac{1}{\varepsilon_{i} \beta_{1-}}-\frac{1}{4 \varepsilon_{i}} .
$$

Solving Equations (39) and (41) together, one can use Equation (33) to obtain their point of intersection:

$$
\delta_{*}^{2}=\frac{\varepsilon_{i}^{2}}{\varepsilon_{1}}\left(1-\frac{\varepsilon_{1}}{\varepsilon_{2}}\right)
$$

which agrees with conditions Equations (36) and (38).

Substituting Equation (42) into Equation (41) leads to an expression for positron energy in the case of high energy initial gamma quanta:

$$
X_{+}=1-X_{-} \approx 1-\frac{1}{4 \varepsilon_{i}}, \quad \varepsilon_{i} \gg 1
$$

As a result, positron energy should not be significantly distinguished from the total energy of the initial gamma quanta. 
For high-energy initial gamma quanta with, for example, $\omega_{1}=45 \mathrm{GeV}$ and $\omega_{2}=55 \mathrm{GeV}$ $\left(\varepsilon_{1}=4.5 \times 10^{2}, \varepsilon_{2}=5.5 \times 10^{2}, \omega_{t h r}=100 \mathrm{MeV}\right)$, one can simply obtain positron energy from Equation (43) $E_{+} \approx 99.975 \mathrm{GeV}$. This can explain the anomalous high-energy positron abundance reported in [3].

In addition to the above-mentioned investigation, there are some studies where the production of fluxes of high-energy positrons [36-38] and gamma quanta [39] near neutron stars was also studied. A study [39] contained the mechanism of high-energy gamma quanta producing to the point of order of $10^{2} \mathrm{GeV}$ near X-ray pulsars.

\section{Resonant Differential Probability}

Resonant differential probability (per time unit) is considered only for channel (a) as far as one for the other channels could be obtained by changing indices, and interference terms are not an object in this work.

The differential probability (per time unit) of the process is defined from amplitude (see Equations (9)-(12)) as follows:

$$
\begin{gathered}
d W=\frac{e^{4}}{4(2 \pi)^{3} E_{-} E_{+} \omega_{1} \omega_{2}}\left|\bar{U}_{p_{-}} M_{0} U_{-p_{+}}\right|^{2} \delta^{(4)}\left(k_{1}+k_{2}-p_{-}-p_{+}\right) d^{3} p_{-} d^{3} p_{+}, \\
M_{0}=\left[\left(\varepsilon_{1}\right)_{\mu} H_{1}^{\mu}\left(q_{-}, p_{-}\right)\right] \frac{\hat{q}_{-}+m}{q_{-}^{2}-m^{2}}\left[\left(\varepsilon_{2}\right)_{v} K_{-1}^{v}\left(q_{-}, p_{+}\right)\right], \\
q_{-}=p_{-}-k_{1}+k=k_{2}+k-p_{+} .
\end{gathered}
$$

Here, the above-mentioned assumption about radiation or absorption of only one photon of the external wave in each vertex is used. After summing up and averaging over initial- and final-state particle polarizations, differential probability takes the following form:

$$
d W=\frac{\alpha^{2} \eta^{4}}{\pi^{3}} C_{1} \frac{G}{\left|q_{-}^{2}-m^{2}\right|^{2}} B_{1} \delta^{(4)}\left(k_{1}+k_{2}-p_{-}-p_{+}\right) d^{3} p_{-} d^{3} p_{+},
$$

where $\alpha$ is the fine structure constant, function $G \sim 1$. Function $C_{1}$ defines the probability of the X-ray-stimulated Compton effect, and function $B_{1}$ defines the probability of the X-ray-stimulated Breit-Wheeler process. These functions also $\sim 1$ and can be written in the following form [27]:

$$
\begin{gathered}
C_{1}=2+\frac{v^{2}}{1+v}-\frac{4 v}{v_{1}}\left(1-\frac{v}{v_{1}}\right), \quad v=\frac{\left(k k_{1}\right)}{\left(k p_{-}\right)}=\frac{\varepsilon_{1}}{\varepsilon_{i} X_{-}}, \quad v_{1}=\frac{2\left(k q_{-}\right)}{m^{2}}=4\left(\varepsilon_{i} X_{-}-\varepsilon_{1}\right) \\
B_{1}=2 u-1+\frac{2 u}{u_{1}}\left(1-\frac{u}{u_{1}}\right), \quad u=\frac{\left(k k_{2}\right)^{2}}{4\left(k p_{+}\right)\left(k q_{-}\right)}=\frac{\varepsilon_{2}^{2}}{4 \varepsilon_{i} X_{+}\left(\varepsilon_{2}-\varepsilon_{i} X_{+}\right)}, \quad u_{1}=\frac{\left(k k_{2}\right)}{2 m^{2}}=\varepsilon_{2},
\end{gathered}
$$

where $v, v_{1}, u, u_{1}$ are kinematic invariants.

The next step is integration with $\delta$-function that leads to the following change:

$$
\delta^{(4)}(\cdots) d^{3} p_{-} d^{3} p_{+} \longrightarrow 2 m^{2} \omega_{i} X_{+}^{2} d \delta_{2+}^{2} d \varphi_{2+}
$$

where $\varphi_{2+}$ is a polar outgoing angle of the positron.

Under the resonance condition, the denominator in Equation (47) turns to zero. To eliminate this infinity, an imaginary additional term to electron mass (Breit-Wigner procedure) is used [9,10]:

$$
m \longrightarrow \mu=m+i \Gamma_{2}, \quad \Gamma_{2}=\frac{q-0}{2 m} W_{2}
$$

where $W_{2}$ is the total probability (per unit time) of the X-ray-stimulated process of electron-positron pair production [27]: 


$$
W_{2}=\eta^{2} \frac{\alpha m^{2}}{8 \pi \omega_{2}} T, \quad T \equiv\left(2+\frac{2}{\varepsilon_{2}}-\frac{1}{\varepsilon_{2}^{2}}\right) \tanh ^{-1}\left(\sqrt{\frac{\varepsilon_{2}-1}{\varepsilon_{2}}}\right)-\left(\frac{\varepsilon_{2}+1}{\varepsilon_{2}}\right) \sqrt{\frac{\varepsilon_{2}-1}{\varepsilon_{2}}} .
$$

After applying substitution Equation (51), the resonant denominator turns into

$$
\left|q_{-}^{2}-\mu^{2}\right|^{2}=16 m^{4}\left[X_{+}^{2}\left(\delta_{2+}^{2}-\delta_{r e s+}^{2}\right)+\frac{4 \Gamma_{2}^{2}}{m^{2}}\right]
$$

where $\delta_{\text {res+ }}^{2}$ is defined by the resonant energy of positron from Equation (21).

After using Equations (50) and (53), differential probability Equation (47) can be rewritten in the following form:

$$
d W_{\text {res }}=\frac{\alpha^{2} \eta^{4}}{8 \pi^{3}} C_{1} \frac{G \omega_{i}}{\left[X_{+}^{2}\left(\delta_{2+}^{2}-\delta_{r e s+}^{2}\right)+Y^{2}\right]} B_{1} X_{+}^{2} d \delta_{2+}^{2} d \varphi_{2+},
$$

where the width of resonance is defined:

$$
\mathrm{Y} \equiv \frac{2 \Gamma_{2}}{m} \sim \alpha \eta^{2}
$$

The denominator in differential probability Equation (54) corresponds to the Breit-Wigner form.

An order of the width of resonance in Equation (54) can be estimated for some typical parameters of an X-ray pulsar. Using Equation (55) for the value of classical parameter $\eta=10^{-1}(\omega=2.5 \mathrm{keV}$, $F \sim 10^{12} \mathrm{~V} / \mathrm{cm}$ ) one can obtain an order $\mathrm{Y} \sim 10^{-4}$. By analogy, for a field with smaller strength and value of parameter $\eta=10^{-2}\left(\omega=2.5 \mathrm{keV}, F \sim 10^{11} \mathrm{~V} / \mathrm{cm}\right)$, the width is of the order of $\mathrm{Y} \sim 10^{-6}$. The obtained values for resonant width are extremely small, which ensures a sufficiently high value of resonant differential probability relative to the nonresonant one.

Equation (54) reaches its maximal value in limit $\delta_{2+}^{2} \rightarrow \delta_{\text {res+ }}^{2}$ :

$$
d W_{r e s}^{\max }=\frac{8}{\pi \alpha^{2} \eta^{4}}\left[\alpha^{2} \eta^{4} C_{1} \frac{G \varepsilon_{2}^{2} \omega_{i}}{T^{2}\left(\varepsilon_{2}-\varepsilon_{i} X_{+}\right)^{2}} B_{1} X_{+}^{2}\right] d \delta_{2+}^{2} d \varphi_{2+} .
$$

Away from resonance, the expression in square brackets in Equation (56) defines the order of differential probability (per unit time) of nonresonant process $d W_{\text {nonres. }}$. Hence, probability for the resonant case can be estimated relatively to the nonresonant case:

$$
d W_{\text {res }}^{\max } \sim \frac{1}{\alpha^{2} \eta^{4}} d W_{\text {nonres }} .
$$

Thus, the result for resonant probability may exceed the nonresonant one by 12 orders of magnitude for an X-ray pulsar with $\omega \sim 1 \mathrm{KeV}$ and $F \sim 10^{11} \mathrm{~V} / \mathrm{cm}$.

\section{Conclusions}

The resonant process of ultrarelativistic electron-positron pair production by ultrarelativistic gamma quanta in the field of an X-ray pulsar was researched, and it has several features.

First, there is the threshold energy for initial gamma quanta due to the $\mathrm{X}$-ray-stimulated Breit-Wheeler process, which is of the order of $10^{2} \mathrm{MeV}$ for the X-ray fields with $F \sim 10^{11}-10^{13} \mathrm{~V} / \mathrm{cm}$.

Second, all particles must be ultrarelativistic and propagate in a narrow cone due to the presence of threshold energy for initial gamma quanta.

Third, there exists strong dependence of energies of final particles on their outgoing angles (see Equations (24) and (29)). Restrictions on outgoing angles were also found (see Equations (25) and (30)). There are two possible values of energies of electron-positron pairs (Figure 3). There is only one possible value for those energies in the case of high-energy initial gamma quanta (see Equations (40) and (43)). 
Lastly, under the resonance condition, an intermediate electron (or positron) state becomes a real particle that leads to resonance infinity in differential probability. This infinity was eliminated by the Breit-Wigner procedure. As a result, the differential probability (per time unit) of the resonant process may exceed the differential probability (per unit time) of the nonresonant case by 12 orders of magnitude for a field near pulsars with $\omega \sim 1 \mathrm{KeV}$ and $F \sim 10^{11} \mathrm{~V} / \mathrm{cm}$. This enormous probability in the case of high-energy initial gamma quanta can explain anomalous positron abundance with energies of about $100 \mathrm{GeV}$ near pulsars [3].

Author Contributions: Conceptualization, S.P.R.; methodology, S.P.R. and V.V.D.; software, V.D.S.; validation, S.P.R., V.V.D. and V.D.S.; formal analysis, S.P.R. and V.D.S.; investigation, S.P.R., V.V.D. and V.D.S.; resources, V.V.D.; data curation, S.P.R. and V.V.D.; writing—original-draft preparation, V.D.S.; writing—review and editing, S.P.R. and V.D.S.; visualization, V.D.S.; supervision, S.P.R. and V.V.D.; project administration, V.V.D.; funding acquisition, V.V.D. All authors have read and agreed to the published version of the manuscript.

Funding: The work was supported by the State program for the fundamental research (theme code FSEG-2020-0024).

Conflicts of Interest: The authors declare no conflict of interest.

\section{References}

1. Khlopov, M. Cosmoparticle physics: The universe as a laboratory of elementary particles. Astron. Rep. 2015, 59, 494-502. [CrossRef]

2. Goldreich, P.; Julian, W.H. Pulsar Electrodynamics. Astrophys. J. 1969, 157, 869. [CrossRef]

3. Hooper, D.; Blasi, P.; Serpico, P.D. Pulsars as the sources of high energy cosmic ray positrons. J. Cosmol. Astropart. Phys. 2009, 2009, 025. [CrossRef]

4. Adriani, O.; Barbarino, G.C.; Bazilevskaya, G.A.; Bellotti, R.; Boezio, M.; Bogomolov, E.A.; Bonechi, L.; Bongi, M.; Bonvicini, V.; Bottai, S.; et al. An anomalous positron abundance in cosmic rays with energies 1.5-100 GeV. Nature 2009, 458, 607-609. [CrossRef]

5. Ruderman, M.A.; Sutherland, P.G. Theory of pulsars: Polar gaps, sparks, and coherent microwave radiation. Astrophys. J. 1975, 196, 51. [CrossRef]

6. Sturrock, P.A. A model of pulsars. Astrophys. J. 1971, 164, 259. [CrossRef]

7. Oleinik, V. Resonance effects in the field of an intense laser beam. J. Exp. Theor. Phys. 1967, $25,697$.

8. Oleinik, V. Resonance effects in the field of an intense laser ray. J. Exp. Theor. Phys. 1968, 26, 1132.

9. Roshchupkin, S.P. Resonant effects in collisions of relativistic electrons in the field of a light wave. Laser Phys. 1996, 6, 837-858.

10. Roshchupkin, S.P.; Padusenko, E.A.; Voroshilo, A.I. Quantum electrodynamics resonances in a pulsed laser field. Laser Phys. 2012, 22, 1113. [CrossRef]

11. Roshchupkin, S.P.; Voroshilo, A.I. Resonant and Coherent Effects of Quantum Electrodynamics in the Light Field; Naukova Dumka: Kiev, Ukraine, 2008.

12. Roshchupkin, S.P.; Lebed, A.A.; Padusenko, E.A.; Voroshilo, A.I. Resonant effects of quantum electrodynamics in the pulsed light field. In Quantum Optics and Laser Experiments; Lyagushyn, S., Ed.; Intech: Croatia, Rijeka, 2012; Chapter 6, pp. 107-156.

13. Roshchupkin, S.P.; Lebed, A.A. Effects of Quantum Electrodynamics in the Strong Pulsed Laser Fields; Naukova Dumka: Kiev, Ukraine, 2013.

14. Voroshilo, A.I.; Roshchupkin, S.P. Resonant scattering of a photon by an electron in the field of a circularly polarized electromagnetic wave. Laser Phys. Lett. 2005, 2, 184-189. [CrossRef]

15. Voroshilo, A.; Roshchupkin, S.; Denisenko, O. Resonance of exchange amplitude of Compton effect in the circularly polarized laser field. Eur. Phys. J. D 2007, 41, 433-440. [CrossRef]

16. Nedoreshta, V.N.; Voroshilo, A.I.; Roshchupkin, S.P. Resonant scattering of an electron by a muon in the field of light wave. Eur. Phys. J. D 2008, 48, 451. [CrossRef]

17. Nedoreshta, V.N.; Voroshilo, A.I.; Roshchupkin, S.P. Resonant scattering of a photon by an electron in the moderately-strong-pulsed laser field. Phys. Rev. A 2013, 88, 052109. [CrossRef]

18. Larin, N.R.; Dubov, V.V.; Roshchupkin, S.P. Resonant photoproduction of high-energy electron-positron pairs in the field of a nucleus and a weak electromagnetic wave. Phys. Rev. A 2019, 100, 052502. [CrossRef] 
19. Doroshenko, D.; Dubov, V.; Roshchupkin, S. Resonant annihilation and production of high-energy electron-positron pairs in an external electromagnetic field. Mod. Phys. Lett. A 2020, 35, 2040023. [CrossRef]

20. Dubov, A.; Dubov, V.V.; Roshchupkin, S.P. Resonant high-energy bremsstrahlung of ultrarelativistic electrons in the field of a nucleus and a weak electromagnetic wave. Laser Phys. Lett. 2020, 17, 045301. [CrossRef]

21. Di Piazza, A.; Müller, C.; Hatsagortsyan, K.Z.; Keitel, C.H. Extremely high-intensity laser interactions with fundamental quantum systems. Rev. Mod. Phys. 2012, 84, 1177. [CrossRef]

22. Ruffini, R.; Vereshchagin, G.; Xue, S.-S. Electron-positron pairs in physics and astrophysics: From heavy nuclei to black holes. Phys. Rept. 2010, 487, 1-140. [CrossRef]

23. Titov, A.I.; Takabe, H.; Kämpfer, B. Nonlinear Breit-Wheeler process in short laser double pulses. Phys. Rev. D 2018, 98, 036022. [CrossRef]

24. Krajewska, K.; Kaminski, J.Z. Breit-Wheeler process in intense short laser pulses. Phys. Rev. A 2012, 86, 052104. [CrossRef]

25. Pustyntsev, A.A.; Dubov, V.V.; Roshchupkin, S.P. Resonant Breit-Wheeler process in an external electromagnetic field. Mod. Phys. Lett. A 2020, 35, 2040027. [CrossRef]

26. Bragin, S.; Di Piazza, A. Electron-positron annihilation into two photons in an intense plane-wave field. arXiv 2020, arXiv:2003.02231.

27. Ritus, V.I.; Nikishov, A.I. Quantum electrodynamics phenomena in the intense field. In Trudy FIAN; Nauka: Moscow, Russia, 1979; Volume 111, pp. 1-276.

28. Bauswein, A.; Just, O.; Janka, H.T.; Stergioulas, N. Neutron-star Radius Constraints from GW170817 and Future Detections. Astrophys. J. 2017, 850, L34. [CrossRef]

29. Walter, R.; Ferrigno, C. X-ray Pulsars. In Handbook of Supernovae; Alsabti, A.W., Murdin, P., Eds.; Springer International Publishing: Cham, Switzerland, 2017; pp. 1385-1399.

30. Volkov, D. On a class of solutions of the Dirac equation. Z. Phys. 1935, 94, 250-260.

31. Wang, H.; Zhong, M.; Gan, L.F. Orthonormality of Volkov Solutions and the Sufficient Condition. Commun. Theor. Phys. 2019, 71, 1179. [CrossRef]

32. Berestetskii, V.B.; Pitaevskii, L.P.; Lifshitz, E.M. Quantum Electrodynamics; Butterworth-Heinemann: Oxford, UK, 1982; pp. 1-652.

33. Schwinger, J. On Gauge Invariance and Vacuum Polarization. Phys. Rev. 1951, 82, 664-679. [CrossRef]

34. Brown, L.S.; Kibble, T.W.B. Interaction of Intense Laser Beams with Electrons. Phys. Rev. 1964, 133, A705-A719. [CrossRef]

35. Roshchupkin, S.P.; Tsybul nik, V.A.; Chmirev, A.N. The Probability of Multiphoton Processes in Quantum-Electrodynamic Phenomena in a Strong Light Field. Laser Phys. 2000, 10, 1256-1272.

36. Sizykh, G.K.; Sergei, P.; Roshchupkin, S.P.; Dubov, V.V. Resonant Ultrarelativistic Electron-Positron Pair Production by High-Energy Electrons in the Field of an X-ray Pulsar. Universe 2020, 6, 132. [CrossRef]

37. Doroshenko, D.V.; Roshchupkin, S.P.; Dubov, V.V. The Resonant Effect of an Annihilation Channel in the Interaction of the Ultrarelativistic Electron and Positron in the Field of an X-ray Pulsar. Universe 2020, 6, 137. [CrossRef]

38. Larin, N.R., Roshchupkin, S.P., Dubov, V.V. Resonant Effects in a Photoproduction of Ultrarelativistic Electron-Positron Pairs on a Nucleus in the Field of the X-ray Pulsar. Universe 2020, 6, 141. [CrossRef]

39. Dubov, A.; Roshchupkin, S.P.; Dubov, V.V. The Resonant Bremsstrahlung of Ultrarelativistic Electrons on a Nucleus with Radiation of Hard Gamma-Quanta in the Presence of a Pulsed Field of the X-ray Pulsar. Universe 2020, 6, 143. [CrossRef]

Publisher's Note: MDPI stays neutral with regard to jurisdictional claims in published maps and institutional affiliations.

(C) 2020 by the authors. Licensee MDPI, Basel, Switzerland. This article is an open access article distributed under the terms and conditions of the Creative Commons Attribution (CC BY) license (http:/ / creativecommons.org/licenses/by/4.0/). 